\title{
Sistema Aerodinámico para un Sistema Eólico de Baja Potencia
}

\section{Aerodynamic System for a Low Power Wind System}

\author{
LINARES-ENRÍQUEZ, Alejandro $\dagger^{* *} \&$ MARTINEZ-ZEPEDA, Ivan
}

Tecnológico de Estudios Superiores de Tianguistenco

ID $1^{\text {er }}$ Autor: Alejandro, Linares-Enríquez / ORC ID: 0000-0002-2250-0730, Researcher ID Thomson: F-8123-21018, CVU CONACYT ID: 607628

ID $1^{\mathrm{er}}$ Coautor: Ivan, Martinez-Zepeda / ORC ID: 0000-0001-7504-7290

DOI: $10.35429 /$ JSI.2019.11.3.19.26

Recibido 20 de Junio, 2019; Aceptado 30 de Septiembre, 2019

\section{Resumen}

Una de los principales necesidades de la sociedad es contar con energía eléctrica en sus hogares, por lo que se desarrolla un mecanismo de orientación del sistema aerodinámico para un Sistema Eólico de Baja Potencia, desarrollando los elementos mecánicos y electrónicos para la orientación de acuerdo a las ráfagas de viento, pero su estructura se encamina a vientos moderados, hasta los $12 \mathrm{~m} / \mathrm{s}$, considerando su aplicación para suministro o si ya cuentan con energía eléctrica para autoabastecimiento, considerando la simulación, el control, el diseño y la implementación del sistema aerodinámico, su forma de orientación mecánica y electrónica, además de determinar cómo influye la incidencia del viento en cada uno de los componentes aerodinámicos del aerogenerador y como les afecta cada uno de los cambios climáticos a los que este se someterá, como son la humedad y la velocidad del viento, como se comporta el sistema dentro de ciertos intervalos del viento en zonas en las que se pueda implementar, por lo que se desarrollaran pruebas en un túnel de viento del comportamiento aerodinámico y se establecerán los elementos del sistema de orientación de las aspas y pruebas de comportamiento dinámico que designaran el material para la fabricación del sistema.

Aerogenerador, Aerodinámica, Baja Potencia

\begin{abstract}
One of the main needs of the society is to have electric power in their homes, which is why an orientation mechanism of the aerodynamic system for a Low Power Wind System is developed, developing the mechanical and electronic elements for orientation according to the gusts of wind, but its development is directed to moderate winds, up to 12 $\mathrm{km} / \mathrm{s}$, considering its application for supply or if they already have electricity for self-supply, considering the simulation, control, design and implementation of the aerodynamic system, its form of mechanical and electronic orientation, as well as determining how the incidence of wind affects each of the aerodynamic components of the wind turbine and how each of the climatic changes to which it is subjected affects them, such as humidity and wind speed, how the system behaves within certain wind intervals in areas in which can be implemented, so that tests in a wind tunnel of aerodynamic behavior will be developed and the elements of the guidance system of the blades and dynamic behavior tests that designate the material for the manufacture of the system will be established.
\end{abstract}

Wind Turbine, Aerodynamics, Low Power

Citación: LINARES-ENRÍQUEZ, Alejandro \& MARTINEZ-ZEPEDA, Ivan. Sistema Aerodinámico para un Sistema Eólico de Baja Potencia. Revista de Innovación Sistemática. 2019. 3-11: 19-26

*Correspondencia al Autor (Correo electrónico: alejandro.linares@ test.edu.mx)

$\dagger$ Investigador contribuyendo como primer Autor. 


\section{Introducción}

En el desarrollo del proyecto se realiza el análisis de los componentes del aerogenerador dentro del diseño, la simulación y la conversión de energía, se definen los parámetros de entrada del sistema, donde la parte aerodinámica es esencial en la obtención de energía, la aerodinámica considera la fuerza que empuja a un objeto en un movimiento relativo, donde se presente la fuerza debida a las diferencias de presiones, definiendo los ángulos de ataque del viento sobre el aspa, con este análisis establecer los elementos mecánicos de orientación para estabilizar el sistema eléctrico de funcionamiento para la obtención de la máxima energía cinética, así como del control de operación y frenado cuando las velocidades sobrepasen los valores establecidos.

México dispone de un potencial renovable indiscutible, con un amplio porfolio de recursos eólicos como los parques eólicos ya instalados. De acuerdo al estudio sobre el potencial eólico, México cuenta con un potencial eólico superior a los $50 \mathrm{GW}$. Esto de acuerdo a lo que comenta el estudio de la Asociación Mexicana de Energía Eólica (AMDEE) y otras entidades, en el plan integral de desarrollo de las energías renovables en México 2013-2018 [1].

La energía eólica es una de las energías renovables que nos brinda los mayores beneficios y posibilidades. Su empleo produce menores impactos en el medio ambiente y a su vez tiene poca afectación por los cambios que el hombre provoca a su entorno.

Dos aplicaciones que ha tenido la energía eólica a través del tiempo son: la navegación de vela y los molinos de viento. La primera de estas aplicaciones es la navegación de vela, donde la orientación determina el mejor aprovechamiento de energía. La otra aplicación son los molinos de viento, donde establece una velocidad lineal que será transformada en velocidad angular, obteniendo un torque y una potencia, que son parámetros indispensables en la aerodinámica del sistema eólico. Los aerogeneradores pueden contribuir significativamente a las necesidades de energía, aunque tengan el nombre de sistemas de baja potencia, las turbinas eólicas son lo suficientemente grandes para proporcionar una parte importante de la energía requerida en los hogares principalmente en las áreas rurales, inmuebles y comercios.
La parte aerodinámica se establece para controlar la velocidad del viento que será transformada en velocidad angular, con un sistema de orientación de las palas que contaran con un anemómetro que permita monitorear la fuerza de sustentación y a ciertos parámetros girar la orientación para disminuir la velocidad con menos área de incidencia, además de contar con un sistema de freno electromecánico.

Nuestra propuesta es determinar la funcionalidad del sistema de orientación de palas para la parte aerodinámica de este tipo de sistemas, determinando sus componentes mecánicos y eléctricos.

\section{Desarrollo}

En el caso de los aerogeneradores debemos monitorear y analizar la potencia incidente del viento, para establecer su control, en las palas analizar cuando el ángulo de torsión es variable, donde se puede utilizar esta variabilidad no sólo para ajustar el ángulo de ataque buscando la potencia máxima sino también para graduar el ángulo de ataque para conseguir una velocidad de rotación constante frente a las diferencias de velocidad del viento incidente.

Para las palas con ángulo de torsión variable, cuando la velocidad incidente del viento es excesiva, para parar el rotor se orienta la pala de manera que entre en pérdidas. En estas condiciones desaparece la fuerza de sustentación que impulsa las palas y el rotor se para.

Estos sistemas por lo general están compuestos por el rotor, multiplicador, freno, generador, cola, torre, cableado, controladores y las baterías, como se muestra en la simulación que permite predecir el comportamiento del sistema de acuerdo a las figuras $1 \mathrm{a}$ y $1 \mathrm{~b}$.

Donde a través del giro de los alabes la turbina convierte la energía cinética del viento en un movimiento rotatorio que acciona el generador eléctrico, se analiza que los sistemas de tres palas son los más eficientes energéticamente de acuerdo a su coeficiente de potencia y su velocidad especifica. 
Para el control aerodinámico determinamos las variables a modificar que son un sistema de regulación por frenos aerodinámico a través de un sistema de orientación de palas, que se activan por la acción de la fuerza centrífuga y que actúa cuando el giro del aspa esta por sobrepasar los límites establecidos de velocidad angular, donde el sistema por medio de palas orientables establecer el ángulo de ataque $\alpha$ de las pala y $\beta$ es la torsión del ángulo de inclinación, de esta forma se consigue variar la fuerza aerodinámica que actúa sobre la pala, variando el ángulo, que se controla por procedimientos mecánicos relacionados con la velocidad de ataque del viento [2].

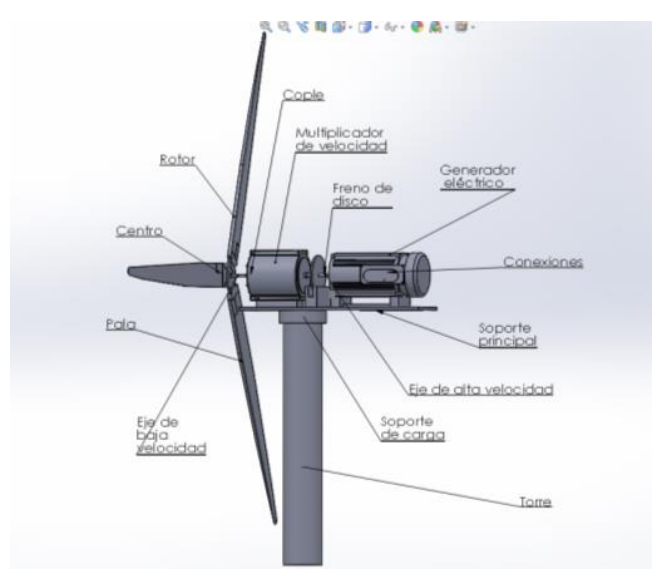

Figura 1a Componentes del Aerogenerador Fuente: Elaboración Propia

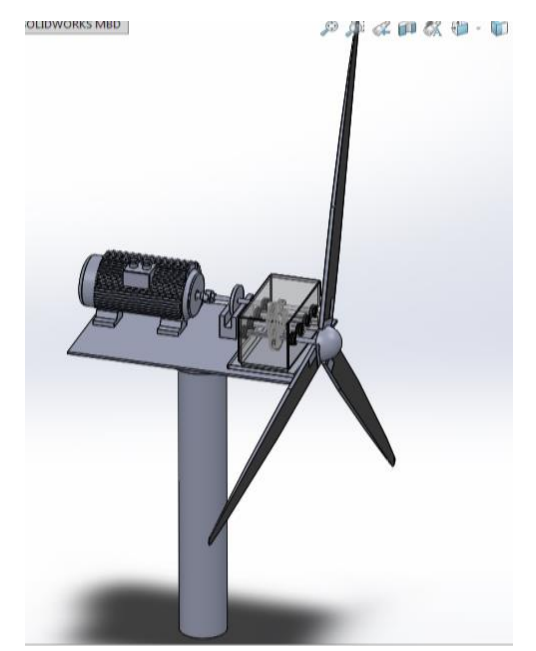

Figura 1b Componentes del Aerogenerador Fuente: Elaboración Propia

\section{Metodología}

La energía cinética del aire moviéndose a una velocidad $v$ está dada por:

$E_{c}=\frac{1}{2} m v^{2}$
Considerando un rotor con un área seccional expuesta a una ráfaga de aire, la energía cinética de la ráfaga de aire disponible para una turbina será:

$E_{c}=\frac{1}{2} \rho_{a} v^{2}$

Una turbina eólica no es capaz de extraer la potencia total que se encuentra en una masa de aire, sino que solo es capaz de extraer una fracción de está. Mediante la ley de la conservación de la masa, el flujo de la masa de aire debe ser igual en todo el tubo de flujo y el gasto másico constante, por lo cual:

$\dot{m}=\rho_{a} A_{1} V_{1}=\rho_{a} A V_{w}=\rho_{a} A_{2} V_{2}$

Donde la variación de la energía cinética del viento por unidad de tiempo es:

$P_{u t i l}=\frac{d\left(E_{c}\right)}{d t}=\frac{1}{2} \rho_{a} A V_{w}\left(V_{1}^{2}-V_{2}^{2}\right)=$
$\rho_{a} A V_{w}^{2}\left(V_{1}-V_{2}\right)$

Estableciendo que la potencia útil máxima que proporciona el rotor considera el coeficiente de potencia que se conoce como el límite teórico de Betz, no es mayor al 59.5\%. [3]. Además, se establece la relación de velocidad específica o periférica, TSR, que es la relación entre la velocidad periférica de la pala $\mathrm{Rw}_{\mathrm{T}}$, y la velocidad del viento $\mathrm{V}_{\mathrm{W}}$, y se encuentra dado por:

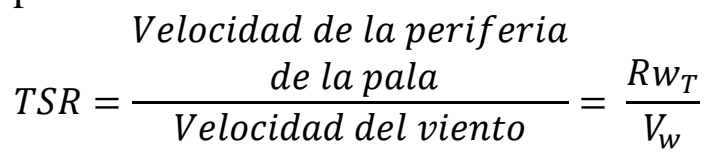

El TSR indica que la periferia de la pala gira a una velocidad TSR mayor que la velocidad del viento $\mathrm{V}_{\mathrm{W}}$ y sirve para comparar el funcionamiento de diferentes máquinas eólicas. El coeficiente de potencia $\mathrm{Cp}$, es la relación entre la potencia que genera la turbina eólica y la energía del viento que atraviesa al rotor:

$C_{p}=\frac{P_{u t i l}}{P}$

El coeficiente de potencia indica la cantidad de energía del viento que se puede convertir en trabajo mecánico por la turbina y es transmitido al eje primario del sistema eólico como potencia mecánica, velocidad angular, torque y esfuerzo mecánico para su análisis. Las gráficas 1a y $1 \mathrm{~b}$ establecen el comportamiento del viento para $9 \mathrm{~m} / \mathrm{s}$ y $15 \mathrm{~m} / \mathrm{s}$ y las características de la potencia.

LINARES-ENRÍQUEZ, Alejandro \& MARTINEZ-ZEPEDA, Ivan. Sistema Aerodinámico para un Sistema Eólico de Baja Potencia. Revista de Innovación Sistemática. 2019 


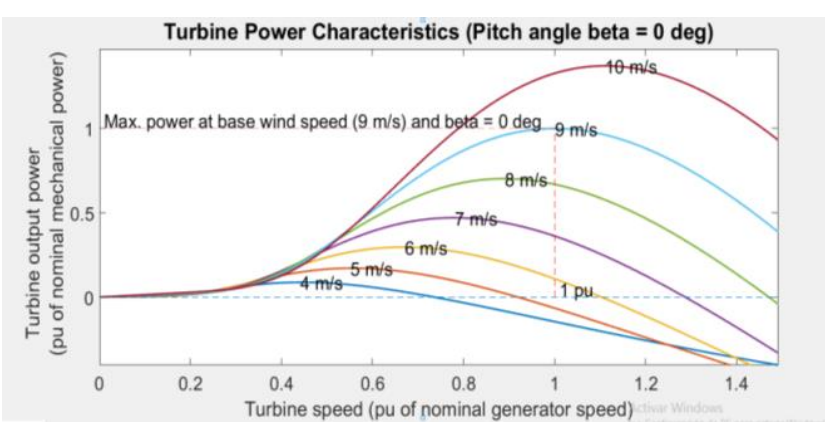

Grafica 1a Características de Potencia Fuente: Elaboración Propia

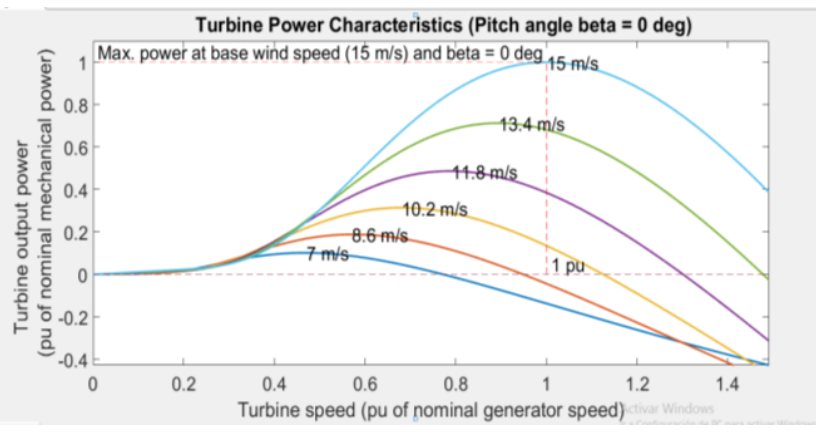

Grafica 1b Características de Potencia Fuente: Elaboración Propia

Dentro del diseño el movimiento de rotación es importante para el análisis del sistema eólico, la ley de Newton de rotación considera la relación entre el par aplicado y su aceleración angular resultante y establece que la suma algebraica de los momentos o pares alrededor de un eje fijo es igual al producto de la inercia por la aceleración angular alrededor del eje, la ecuación es:

$\sum T=J \propto=J \frac{d w}{d t}=J \frac{d^{2} \theta}{d t^{2}}$

Donde $\mathrm{J}$ es la inercia, $\propto$ es la aceleración angular, $\mathrm{T}$ es el par, w es la velocidad angular, $\theta$ es el desplazamiento angular, en el que el par $\mathrm{T}$ es la fuerza de torsión que se transmite y se ejerce sobre los ejes y engranes, el par de torsión depende de la magnitud de la fuerza aplicada y de la distancia entre el eje de rotación y la línea de acción de la fuerza. Además dentro de la torsión el análisis del ángulo de giro en radianes de los ejes se calcula mediante el análisis torsional de la ley de Hooke $\tau=G \gamma$ y $\gamma=\rho \theta / L$ estableciendo el torque como una fuerza por una distancia: $T=\int \rho(\tau d A)$ y estableciendo a $\int \rho^{2} d A=J$ que es el momento polar de inercia, obteniendo el ángulo de torsión en radianes, donde estos elementos son importantes para el desarrollo del sistema $[4,5,6,7]$.
$\theta=\frac{T L}{G J}$

Donde:

$\mathrm{T}=$ Torsión.

$\mathrm{L}=$ Longitud.

$\mathrm{G}=$ Modulo de Rigidez.

$\mathrm{J}=$ Segundo Momento Polar de Inercia.

De acuerdo a este análisis tenemos dos tipos de cargas que actúan sobre el rotor, son la fuerza centrífuga que es una carga estática perpendicular al eje del giro, proporcionando una componente de tensión a lo largo de la pala y otra de flexión en sentido contrario al de las cargas aerodinámicas $\mathrm{y}$, las cargas dinámicas son debidas al giro de la pala, existiendo también cagas transitorias debido a la operación del sistema.

\section{Sistema Aerodinámico del Sistema Eólico}

La regulación por medio de palas orientables consiste en analizar y diseñar en base al ángulo de ataque $\alpha$ de las pala con la velocidad del viento y el ángulo $\beta$ que es la torsión del ángulo de inclinación y de esta forma conseguir variar la fuerza aerodinámica, de acuerdo a la pala para la captación de la energía cinética, se tendrá el tamaño de las palas de 3 metros para el proyecto, el perfil y los elementos de unión de la pala, con el diseño aerodinámico que al orientarse cambiara la intensidad de incidencia del viento, en el cual se obtiene la simulación con la caracterización del perfil, la geometría y el modelado.

La energía eólica establece los análisis de las palas del sistema eolico, como se muestra en la figura $2 \mathrm{a}$ y $2 \mathrm{~b}[8,9]$.

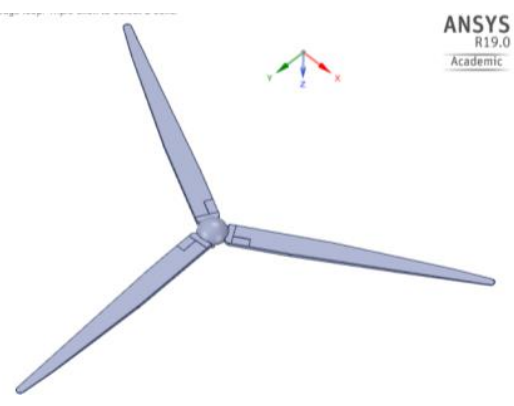

Figura 2a Aspas Aerogenerador Fuente: Elaboración Propia 


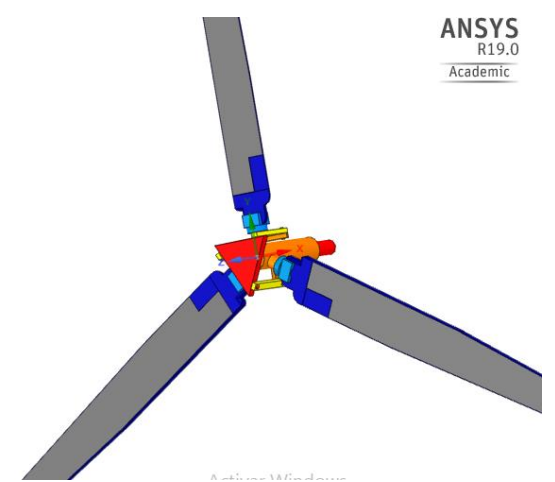

Figura 2b Aspas Aerogenerador Fuente: Elaboración Propia

El desarrollo de la parte aerodinámica considera la estructura de las palas en el diseño aerodinámico, estableciendo una longitud de tres metros para el proyecto, con un diseño mecánico herramental en el cual podemos tener un soporte plano o un soporte cilíndrico para la ubicación de la pala en el sistema de giro, para que la incidencia sea mayor o menor, se debe considerar un diseño optimo y las posibles modificaciones geométricas y constructivas de acuerdo a un estado de cargas.

Se debe establecer un diseño de modelos para la geometría de las palas a implementar y, poderlo desarrollar dentro del sistema eólico de baja potencia, también se debe establecer el desarrollo de los métodos de fabricación, pruebas en materiales como lo son alguna resina, acrílico o espuma.

Con estos modelos establecer las velocidades del viento para su aplicación en un túnel de viento, como se muestra en la figura 3, Se debe analizar el coeficiente de empuje del sistema y los coeficientes de las palas que son la densidad, la velocidad relativa y la longitud de la pala para establecer el coeficiente de potencia.

\footnotetext{
Además para determinar el comportamiento de la estabilidad de la orientación de las palas se desarrolló un análisis de estabilidad donde se observa el comportamiento considerando las variables que son el viento incidente y sus cambios, el ángulo de ataque y el ángulo de inclinación, para lograr a través de las características del aerogenerador establecer la relación de entrada que el viento tiene con la salida que es la energía eléctrica, y dentro del sistema establecer el comportamiento de velocidad angular, torque y potencia, que pasara a voltaje y amperaje, para finalizar en potencia eléctrica, como se muestra en la figura $4[10,11]$.
}

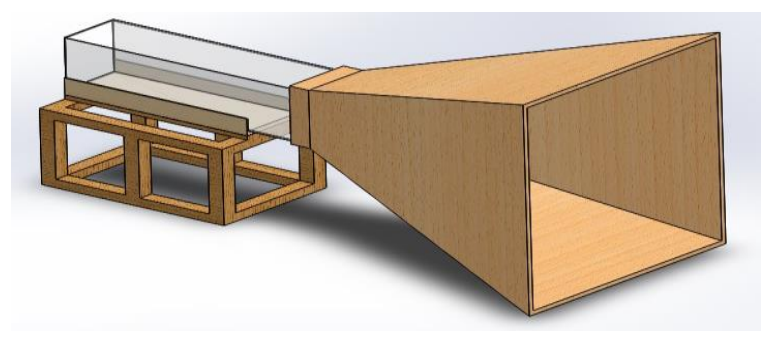

Figura 3 Túnel de viento Fuente: Elaboración Propia

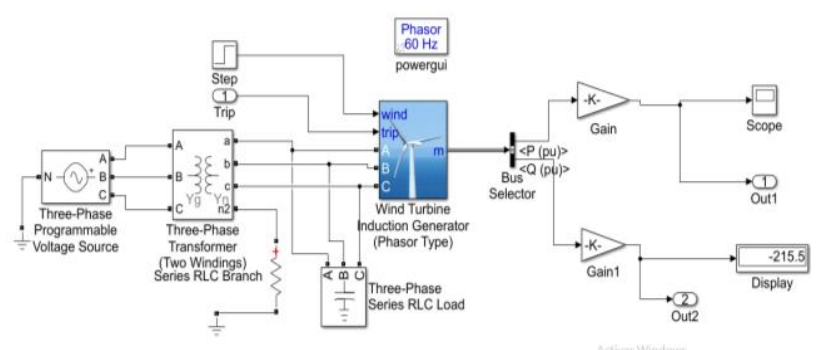

Figura 4 Sistema de Estabilidad Fuente: Elaboración Propia

Donde la estabilidad se logra primeramente en 0.2 segundo y se logra totalmente en 1.6 segundos, como se muestra en la gráfica 2 , considerando una velocidad de 12 $\mathrm{m} / \mathrm{s}$.

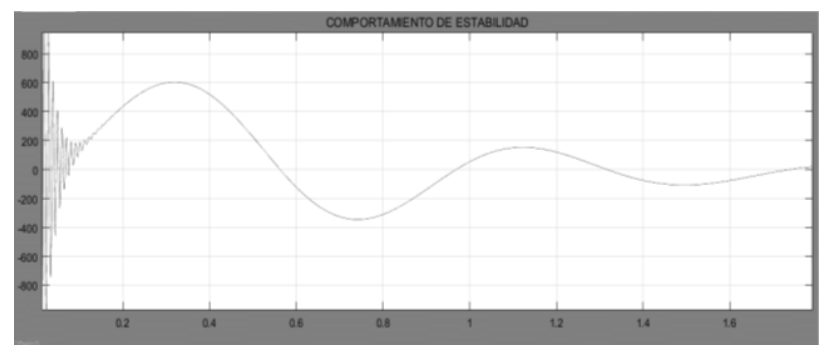

Grafica 2 Comportamiento de Estabilidad Fuente: Elaboración Propia

El sistema de estabilidad se analiza en la construcción de un túnel de viento ya que se pueden hacer variar la velocidad y establecer la simulación a condiciones reales de velocidades del viento, para las condiciones aerodinámicas que se analizaran al aspa y su comportamiento de acuerdo a la orientación que se tenga, el túnel de viento que se desarrolló como se muestra en la figura 5 , con un área de $2500 \mathrm{~cm}^{2}$ para un aspa de prueba de $22 \mathrm{~cm}$ de radio. 


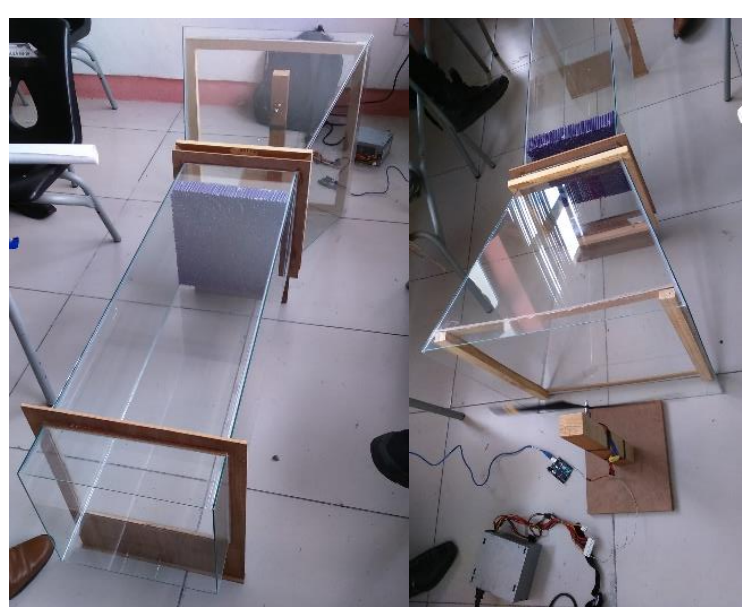

Figura 5 Túnel de Viento

Fuente: Elaboración Propia

\section{Resultados}

Para el sistema eólico de baja potencia se desarrolló un sistema mecánico de orientación de las palas, en el cual contribuya a establecer los elementos para que se pueda controlar la velocidad, potencia y torque en el sistema, esta propuesta establece una simulación mecánica de cómo se orientara las palas en el sistema, como se muestra en la figura 6 .

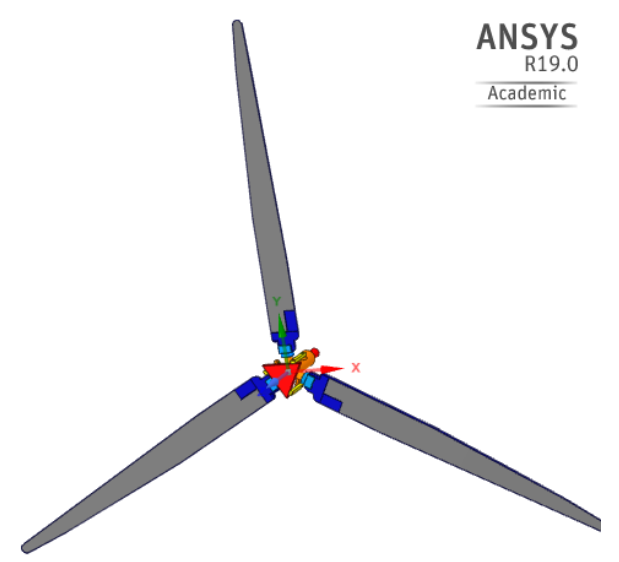

Figura 6 Mecanismo de Orientación de Aspas Fuente: Elaboración Propia

Con el análisis del sistema aerodinámico se establecieron los elementos para orientación de las palas el cual a una velocidad de $9 \mathrm{~m} / \mathrm{s}$ inicia la inclinación desde los cero grados, llegando a los $15 \mathrm{~m} / \mathrm{s}$ como velocidad máxima, donde la orientación sea a 90 grados para disminuir al máximo el área de incidencia.

El equipo además contara con un sistema de frenado electromecánico para que apoye al aerogenerador en condiciones de mayores vientos y que no provoque un daño al equipo, monitoreando la velocidad del viento que se presente en la zona de instalación y prueba.
El sistema de orientación cuenta con un mecanismo de un eje tubular principal y salientes a 120 grados para que las palas puedan girar de acuerdo a la incidencia del viento, cuenta con un mecanismo de salida de otro eje central que moverá el eje tubular de acuerdo a la velocidad en una relación de diámetro con distancia longitudinal, estableciendo el ángulo en el cual debe estar girado el sistema, como se muestra en la figura 7 a y $7 b,[8,9]$. El sistema tiene una relación lineal de acuerdo a la capacidad de giro que tienen las aspas.

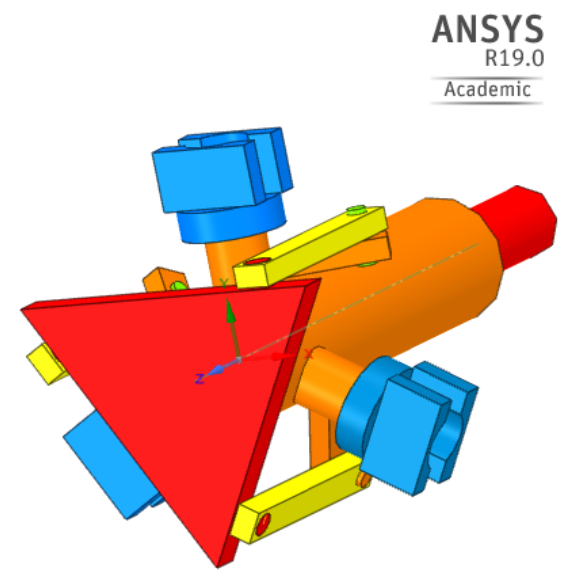

Figura 7a Sistema de Orientación Fuente: Elaboración Propia

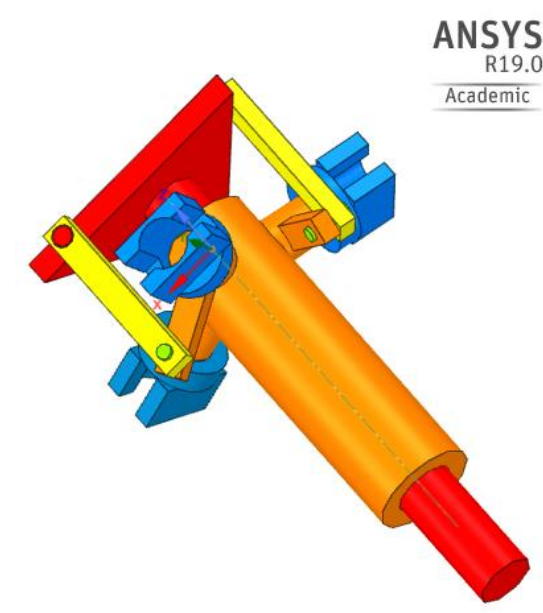

Figura 7b Sistema de Orientación Fuente: Elaboración Propia

Las partes del sistema establecen la estructura donde serán colocadas las aspas del aerogenerador, esta base de las palas al contar con un sistema de barras el cual se orienta, ara girar las palas, además la barra de transmisión se encuentra unida a una delta que es el elemento central del sistema orientador en la parte aerodinámica del sistema eólico. 
Con el análisis del sistema aerodinámico se está iniciando la construcción del sistema de orientación el cual este acoplado a un sistema de engranajes como parte del sistema mecánico, como se muestra en la figura $8 \mathrm{a}$ y $8 \mathrm{~b}$, para pasar posteriormente a un generador de imanes permanentes, el sistema de orientación permite mejor el aprovechamiento de la energía cinética del viento para su transformación en energía mecánica y finalizar con transformación en energía eléctrica.

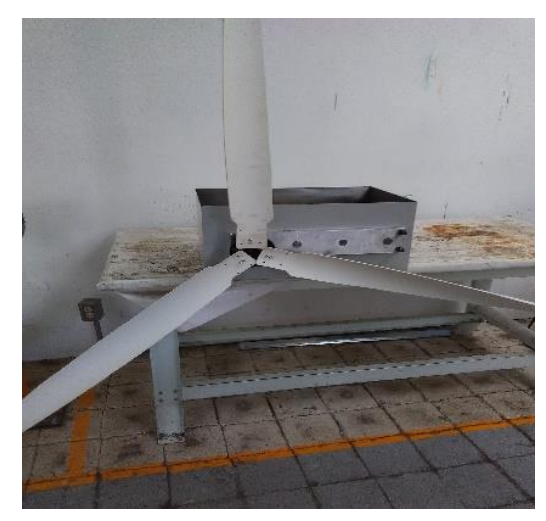

Figura 8a Sistema Eolico en construcción, inicio desarrollo de sistema de orientación

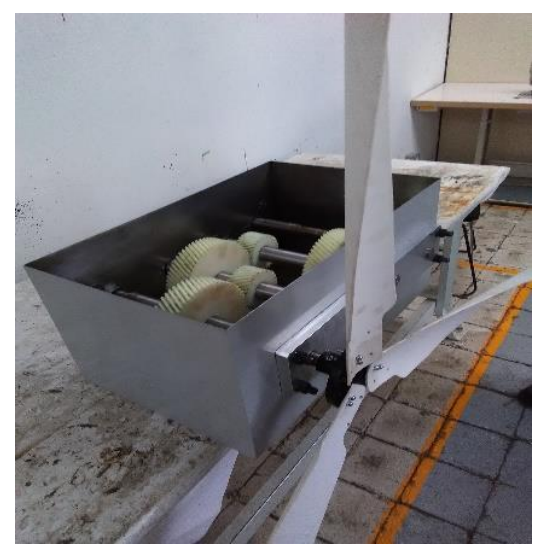

Figura 8b Sistema Eolico en construcción, inicio desarrollo de sistema de orientación

Los resultados del sistema de orientación establecen que se determina un sistema mecánico de ensamble y engranaje en la orientación del equipo para que se realicen las pruebas con un túnel de viento a escala y, poder potenciar estos resultados a un sistema que se montará para su comportamiento en la región del Estado de México, esto dentro del desarrollo de la investigación en el comportamiento eólico y aprovechamiento de este tipo de energía renovable.

\section{Agradecimiento}

Al Tecnológico de Estudios Superiores de Tianguistenco el poder desarrollar el diseño, la simulación y propuesta de desarrollo del sistema aerodinámico del sistema eólico de baja potencia.

\section{Conclusiones}

La simulación del sistema eólico permite establecer los elementos de análisis en su parte aerodinámica para el aprovechamiento de las ráfagas del viento y conocer las variables que serán importantes en la transformación de energía mecánica a energía eléctrica.

El sistema aerodinámico es un elemento de captación y control del equipo, por lo que su desarrollo es de suma importancia para que estos no colapsen o puedan presentar fallas, generando propuestas que permitan no solamente el mejor aprovechamiento del recurso eólico, sino también su control y estabilidad.

El análisis y diseño de los componentes que formaran el sistema de orientación son sistemas que deben ser analizados y evaluados, ya que podemos tener diferentes propuestas para mejorar estos mecanismos que ayuden a la aerodinámica de los sistemas eólicos de baja potencia.

El proyecto establece, desarrolla y muestra la importancia del diseño, la simulación y el control dentro de la ingeniería para los sistemas eólicos, por lo que los resultados obtenidos demuestran el funcionamiento y la operación del aerogenerador.

\section{Referencias}

[1]. PwC en colaboración con Climate Works Foundation, Iniciativa Mexicana para las Energías Renovables (IMERE) y el World Wildlife Fund (WWF), Plan integral para el desarrollo de las energías renovables en México 2013-2018 Propuesta de escenarios y acciones necesarias para su desarrollo.

[2] Villarrubia Lopez Miguel, (2012) Ingeniería de la Energía Eólica, 1ra. Edición, Alfaomega. 
[3]. Raúl Garduño Ramírez, Marino Sánchez Parra, Roberto Hernández González, (2012), Investigación, Desarrollo e Innovación Tecnológica de Sistema del Control de Aerogeneradores, Boletín IIE, Abril - Junio 2012.

[4]. Richard G. Budynas y J. Keith Nisbett. (2012), Diseño de Ingeniería Mecánica de Shigley, Ed. Mc Graw Hill, 9na Edición.

[5] Ferdinand P.Beer, E Russell Johnston, Jr. (2004) Mecánica de Materiales, Tercera edición, Mc Grall Hill.

[6] Ferdinand P. Beer, E Russell Johnston, Jr. (2007), Mecánica Vectorial para Ingenieros, Octava edición, Mc Grall Hill.

[7] Hamilton H. Mabie, Charles F. Reinholtz, (2002) Mecanismos y Dinámica de Maquinaria, ed. Limusa Wiley, 2da edición.

[8]. Ansys, Inc. Manual ANSYS 18.2, 2017.

[9]. Ansys, Inc. ANSYS FLUENT USER'S GUIDE, Release 13.0, November 2010.

[10]. Amos Gilat, MATLAB. Ed. Reverte, 2005.

[11]. Enrique Pino Bermudes, Fernando Matía Espada, Fundamentos de Control con MATLAB. Ed. Pearson, 2012. 